\title{
INEQUALITIES FOR THE GENERALIZED TRIGONOMETRIC, HYPERBOLIC AND JACOBIAN ELLIPTIC FUNCTIONS
}

\author{
EDWARD NEUMAN
}

Abstract. This paper deals with the inequalities for the generalized trigonometric, hyperbolic and the Jacobian elliptic functions. These families of higher transcendental functions are of great importance in the studies of some problems that arose in the theory of differential equations. Among the main results established in this paper the Wilker- and Huygens- type inequalities for the functions under discussion are obtained.

Mathematics subject classification (2010): 26D05, 33E30, 33E05, 26E60.

Keywords and phrases: Generalized trigonometric, hyperbolic and Jacobian elliptic functions, $R$ hypergeometric functions, means, Gauss hypergeometric function, inequalities.

\section{REFERENCES}

[1] A. BARICZ, B. A. BHAYO, R. KLÉN, Convexity properties of generalized trigonometric and hyperbolic functions, Aequat. Math. (2013), doi:10.1007/s00010-013-0222-x.

[2] B. A. BнAYo, Power mean inequality of generalized trigonometric functions, preprint.

[3] B. A. Bhayo, M. Vuorinen, On generalized trigonometric functions with two parameters, J. Approx. Theory 164 (2012), No. 10, 1415-1426.

[4] B. A. BHAYO, M. VUORINEn, Inequalities for eigenfunctions of the p-Laplacian, Issues of Analysis 2 (20) (2013), No. 1, 13-35.

[5] J. M. Borwein, P. B. Borwein, Pi and the AGM - A Study in Analytic Number Theory and Computational Complexity, Wiley, New York, 1987.

[6] J. BRenner, B. C. CARLson, Homogeneous mean values: Weights and asymptotics, J. Math. Anal. Appl. 123 (1987), 265-280.

[7] B. C. CARlson, A hypergeometric mean value, Proc. Amer. Math. Soc. 16 (1965), 759-766.

[8] B. C. CARLson, Some inequalities for hypergeometric functions, Proc. Amer. Math. Soc. 17 (1966), 32-39.

[9] B. C. CARLson, Algorithms involving arithmetic and geometric means, Amer. Math. Monthly 78 (1971), 496-505.

[10] B. C. Carlson, Special Functions of Applied Mathematics, Academic Press, New York, 1977.

[11] P. DRÁBEK, R. MANÁSEVICH, On the closed solution to some p-Laplacian nonhomogeneous eigenvalue problem, Differential Integral Equations 12 (1999), 773-788.

[12] D. E. Edmunds, P. Gur Ka, J. LANG, Properties of generalized trigonometric functions, J. Approx. Theory 164 (2012), 47-56.

[13] C. Huygens, Oeuvres Completes 1888-1940, Société Hollandaise des Science, Haga.

[14] W.-D. Jiang, M.-K. Wang, Y.-M. Chu, Y.-P. JiAng, F. QI, Convexity of the generalized sine function and the generalized hyperbolic sine function, J. Approx. Theory 174 (2013), 1-9.

[15] R. KlÉN, M. Visuri, M. VuORInEN, On Jordan type inequalities for hyperbolic functions, J. Inequal. Appl. (2010), Article ID 362548, 14 pp.

[16] R. KLÉN, M. VUORINEN, X. ZHANG, Inequalities for the generalized trigonometric and hyperbolic functions, J. Math. Anal. Appl. 409 (2014), 521-529.

[17] P. LindQvist, Some remarkable sine and cosine functions, Ricerche di Matematica 44 (1995), No. 2, 269-290.

[18] D. S. Mitrinović, Analytic Inequalities, Springer-Verlag, Berlin, 1970. 
[19] E. Neuman, On Gauss lemniscate functions and lemniscatic mean, Math. Pannon. 18 (2007), 77-94.

[20] E. NEUMAn, One-and two-sided inequalities for Jacobian elliptic functions and related results, Integral Transforms Spec. Funct. 21 (2010), 399-407.

[21] E. NEUMAn, Inequalities for the Schwab-Borchardt mean and their applications, J. Math. Inequal. 5 (2011), No. 4, 601-609.

[22] E. Neuman, Inequalities and bounds for generalized complete elliptic integrals, J. Math. Anal. Appl. 373 (2011), 203-213.

[23] E. Neuman, Some inequalities for the gamma function, Appl. Math. Comput. 218 (2011), 43494352.

[24] E. Neuman, Inequalities for weighted sums of powers and their applications, Math. Inequal. Appl. 15 (2012), No. 4, 995-1005.

[25] E. Neuman, On Gauss lemniscate functions and lemniscatic mean II, Math. Pannon. 23 (2012), 6573.

[26] E. NEUMAN, Inequalities for Jacobian elliptic functions and Gauss lemniscate functions, Appl. Math. Comput. 218 (2012), 7774-7782.

[27] E. NEuman, Product formulas and bounds for Jacobian elliptic functions with applications, Integral Transforms Spec. Funct. 23 (2012), 347-354.

[28] E. Neuman, On lemniscate functions, Integral Transforms Spec. Funct. 24 (2013), 164-171.

[29] E. Neuman, A note on the Jacobian elliptic sine function, Integral Transforms Spec. Funct. 24 (2013), $548-553$.

[30] E. NEUMAN, Wilker and Huygens-type inequalities for the generalized trigonometric and for the generalized hyperbolic functions, Appl. Math. Comput. 230 (2014), 211-217.

[31] E. NEUMAN, Wilker and Huygens-type inequalities for Jacobian elliptic and theta functions, Integral Transforms Spec. Funct. 25 (2014), 240-248.

[32] E. NEUMAN, Inequalities involving generalized trigonometric and generalized hyperbolic functions, J. Math. Inequal. 8 (2014), No. 4, 725-736.

[33] E. Neuman, On the p-version of the Schwab-Borchardt mean, Internat. J. Math. Math. Sci., Volume 2014, Article ID 697643, 7 pages.

[34] E. Neuman, On the inequalities for the generalized trigonometric functions, Internat. J. Anal., Volume 2014, Article ID 319837, 5 pages.

[35] E. Neuman, J. SÁndor, On the Schwab-Borchardt mean, Math. Pannon. 14 (2003), No. 2, $253-266$.

[36] E. Neuman, J. SÁndor, On the Schwab-Borchardt mean II, Math. Pannon. 17 (2006), No. 1, 49-59.

[37] E. NEUMAN, J. SÁNDOR, On some inequalities involving trigonometric and hyperbolic functions with emphasis on the Cusa-Huygens, Wilker and Huygens inequalities, Math. Inequal. Appl. 13 (2010), No. 4, 715-723.

[38] E. NEUMAN, J. SÁNDOR, Inequalities involving Jacobian elliptic functions and their inverses, Integral Transforms Spec. Funct. 23 (2012), 719-722.

[39] F. W. J. Olver, D. W. Lozier, R. F. Boisvert, C. W. Clark (Eds.), NIST Handbook of Mathematical Functions, Cambridge Univ. Press, New York, 2010.

[40] J. E. PeČarić, F. Proschan, Y. I. Tong, Convex Functions, Partial Orderings and Statistical Applications, Academic Press, Boston, 1992.

[41] S. TAKEUCHI, Generalized Jacobian elliptic functions and their application to bifurcation problems associated with p-Laplacian, J. Math. Anal. Appl. 385 (2012), 24-35.

[42] S. TAKEUCHI, The basis property of generalized Jacobian elliptic functions, arXiv math.CA $1310.0597 \mathrm{v} 1$.

[43] J. B. Wilker, Problem E 3306, Amer. Math. Monthly 96 (1989), 55.

[44] S. WU, A. BARICZ, Generlizations of Mitrinović, Adamović and Lazarevic's inequalities and their applications, Publ. Math. Debrecen 75(2009), No. 3-4, 447-458.

[45] S.-H. WU, H. M. SRIVASTAVA, A weighted and exponential generalization of Wilker's inequality and its applications, Integral Transform. Spec. Funct. 18 (2007), No. 8, 525-535.

[46] L. ZHU, A new simple proof of Wilker's inequality, Math. Inequal. Appl. 8 (2005), No. 4, 749-750.

[47] L. ZHU, On Wilker-type inequalities, Math. Inequal. Appl. 10 (2007), No. 4, 727-731.

[48] L. ZHU, Some new Wilker type inequalities for circular and hyperbolic functions, Abstract Appl. Analysis, Vol. 2009, Article ID 485842, 9 pages. 